\title{
Socially Embedded Collaborative Practices: Introduction to a Special Issue based on the COOP 2010 Conference
}

\author{
Myriam Lewkowicz ${ }^{1} \&$ Markus Rohde ${ }^{2}$ \\ ${ }^{1}$ ICD/Tech-CICO UMR CNRS 6279, Troyes University of Technology (UTT), 12, rue Marie Curie, \\ BP 2060, 10010 Troyes Cedex, France (E-mail: myriam.lewkowicz@utt.fr); ${ }^{2}$ Information Systems \\ and New Media, University of Siegen, Hoelderlinstr. 3, 57068 Siegen, Germany (E-mail: markus. \\ rohde@uni-siegen.de)
}

This special issue focuses on socially embedded collaborative practices in different domains, based on the 9th International COOP Conference, which took place in Aix-en-Provence in May, 2010 and was documented in the conference proceedings (Lewkowicz et al. 2010).

We present four case studies on the support of collaborative technologies for socially relevant tasks in the fields of healthcare, political engagement, science, and open source software development: software development of IT systems in the healthcare sector (Federico Cabitza), the use of collaborative technology by social movements (Saqib Saeed, Markus Rohde, and Volker Wulf), the use of technology and artefacts in archaeology (Marco Locatelli, Carla Simone, and Viviana Ardesia), and the use of the Eclipse development platform among software engineers (Gunnar Stevens and Sebastian Draxler). All of these studies apply an ethnographic research approach and strive to achieve a deep understanding of the collaborative practices in these domains and their respective social context in order to derive specific design requirements for appropriate technological support.

In addition to the case studies, this issue presents a theoretical and programmatic paper focusing on the concept of "work" in CSCW. Kjeld Schmidt contributes to an ongoing debate on the scope of CSCW research, responding to an article by Andy Crabtree et al. (2005) that argues for a broadened perspective of CSCW. Schmidt's contribution reflects on conceptual issues as well as the socio-economic status of "work" and warns CSCW scholars against abandoning the discipline's central research focus. The issues raised in this position paper, together with issues raised by Schmidt at ECSCW 2009 (Schmidt, 2009), led Myriam Lewkowicz and Volker Wulf, the scientific chairs of COOP 2010, to 
organize a panel on "the future of CSCW", with Kari Kuutti, University of Oulu, Finland; Gary Olson, University of California, Irvine, USA; Kjeld Schmidt, Copenhagen Business School, Denmark; and Volker Wulf, University of Siegen, Germany. Focusing on questions such as how to define CSCW as a field of research, the alleged 'fragmentation' of the field, the methodological implications of focusing on collaborative practices, etc., the resulting debate was both lively and fruitful.

The International Conference Series on the Design of Cooperative Systems (COOP conference) is one of the most important gatherings of CSCW researchers in Europe. It was launched 18 years ago by the French COOP group, now replaced by the COOP steering committee, consisting of some leading European scholars in this discipline: Liam Bannon, Françoise Darses, Gorgio De Michelis, Alain Giboin, Parina Hassanaly, Thomas Herrmann, Myriam Lewkowicz, Pascal Salembier, Kjeld Schmidt, Carla Simone, Wolfgang Prinz, Volker Wulf, and Manuel Zacklad.

During the past 18 years, has COOP undergone a thematic development and is now considered the second European conference on Computer Supported Cooperative Work. The COOP conference series is supported by the European Society of Socially Embedded Technologies (EUSSET) and is held every second year alternately with the European Conference on Computer Supported Cooperative Work (ECSCW), usually at the French Riviera. COOP conferences recognize that cooperative systems design requires a deep understanding of collective activities, involving both artifacts and social practices, and contributions are thus solicited from a wide range of research areas contributing to the fields of cooperative systems design and evaluation, such as Computer Supported Cooperative Work, Human Computer Interaction, Participatory Design, Information Systems, Knowledge Management, Ethnography, Organizational and Management Sciences, Sociology, Psychology, and Linguistics.

Focusing on socially embedded collaborative practices, the editors selected Schmidt's programmatic contribution and the four case studies and the authors were invited to reconsider and extend their COOP papers for this special issue. All contributions underwent an additional blind peer-reviewing procedure and were revised by the authors according to the reviewers' requests.

The guest editors of this issue would like to thank the editors of the CSCW Journal for the opportunity to publish this selection of positions and studies on socially embedded collaborative practices and to contribute to the further development of $\mathrm{CSCW}$ research. We thank all COOP conference participants, all involved reviewers and the authors for their contributions. We hope that we succeeded in providing an interesting and significant selection of research contributions to the field of CSCW and the respective discourse in Europe.

August 2011, Myriam Lewkowicz and Markus Rohde 


\section{References}

Crabtree, A., Rodden, T. A., \& Benford, S. D. (2005). Moving with the times: IT research and the boundaries of CSCW, Computer Supported Cooperative Work (CSCW). The Journal of Collaborative Computing, 14, 217-251.

Lewkowicz, M., Hassanaly, P., Rohde, M., \& Wulf, V. (2010). Proceedings of COOP 2010, CSCW Book Series. London: Springer.

Schmidt, K. (2009). Divided by a common acronym: On the fragmentation of CSCW. In I. Wagner et al. (Eds.), ECSCW 2009: Proceedings of the 11th European Conference on ComputerSupported Cooperative Work, 7-11 September 2009 (pp. 223-242). Vienna, Austria, London: Springer. 